\title{
THE DUNFORD-PETTIS PROPERTY IN THE PREDUAL OF A VON NEUMANN ALGEBRA
}

\author{
L. J. BUNCE
}

(Communicated by Palle E. T. Jorgensen)

\begin{abstract}
The von-Neumann algebras whose predual has the Dunford-Pettis property are characterised as being Type I finite. This answers the question asked by Chu and Iochum in The Dunford Pettis property in $C^{*}$-algebras, Studia Math. 97 (1990), 59-64.
\end{abstract}

The purpose of this paper is to characterise those von Neumann algebras $M$ for which the predual $M_{*}$ has the Dunford-Pettis property thereby settling the question raised in [1].

A Banach space $X$ is said to have the Dunford-Pettis property if for each Banach space $Y$ each weakly compact linear operator from $X$ to $Y$ sends weakly convergent sequences to norm convergent sequences. Classically, all $L_{1}$ spaces (Dunford and Pettis) and all $C(X)$ spaces (Gröthendieck) have the Dunford-Pettis property (see [2] for much more). A thorough study of the Dunford-Pettis property in $C^{*}$-algebras and von Neumann algebras was undertaken in [1] and [3], to which we refer the reader for any unmentioned details. But one question remained unanswered. It was proved in [1] and [3] that if $M$ is a von Neumann algebra then

(a) if $M_{*}$ has the Dunford-Pettis property then $M$ is finite;

(b) if $M$ is Type I finite then $M_{*}$ has the Dunford-Pettis property.

An obstacle to a characterisation was that it was not known whether it was possible for the predual of a Type $\mathrm{II}_{1}$ von Neumann algebra to have the Dunford-Pettis property. We show that is it not possible. Thus we establish the following.

Theorem. The following are equivalent for a von Neumann algebra $M$.

(i) $M_{*}$ has the Dunford-Pettis property.

(ii) $M$ is Type I finite.

Proof. In order to establish the validity of the converse of (b) let $M$ be a von Neumann algebra for which $M_{*}$ has the Dunford-Pettis property. In view of (a), and since the predual of every summand of $M$ clearly inherits the property, it can be supposed that $M$ is of Type $\mathrm{II}_{1}$. At this point we appeal to the Jordan operator theory of spin factors contained in [4-6]. Since $M$ contains a

Received by the editors October 31, 1990 and, in revised form, February 9, 1991.

1980 Mathematics Subject Classification (1985 Revision). Primary 46L05, 46L10, 46B20.

(C) 1992 American Mathematical Society $0002-9939 / 92 \$ 1.00+\$ .25$ per page 
Type $\mathrm{II}_{1}$ subfactor it certainly contains a countably infinite spin system $\left\{s_{n}\right\}$, consisting of nontrivial symmetries $s_{n}$ (in $M_{s a}$ ) satisfying $s_{n} s_{m}+s_{m} s_{n}=0$ whenever $n \neq m$. The real Banach subspace $V$ of $M_{s a}$ generated by $\left\{s_{n}\right\}$ is a JW-subalgebra of $M_{s a}$ (called a spin factor). In addition, $V$ is an infinite dimensional real Hilbert space in an equivalent norm. Evidently, then, there exists a sequence $\left(x_{n}\right)$ in $V$ such that $\left\|x_{n}\right\|=1$ for all $n$ and $x_{n} \rightarrow 0$ in the $\sigma\left(V, V^{*}\right)$ topology. Clearly $x_{n} \rightarrow 0$ in the $\sigma\left(M, M^{*}\right)$ topology. But as $M_{*}$ has the Dunford-Pettis property this means that $x_{n}^{2} \rightarrow 0$ in the $\sigma\left(M, M_{*}\right)$ topology, by [3, Lemma 1] or [1, Corollary 5]. Hence $x_{n} \rightarrow 0$ in the strong operator topology. But as proved in [5, Theorem 7.1] the latter coincides on $V$ with the norm topology. So $x_{n} \rightarrow 0$ in norm, a contradiction which completes the proof.

\section{REFERENCES}

1. C.-H. Chu and B. Iochum, The Dunford Pettis property in $C^{*}$-algebras, Studia Math. 97 (1990), 59-64.

2. J. Diestel, A survey of results related to the Dunford-Pettis property, Contemp. Math., vol. 2, Amer. Math. Soc., Providence, RI, 1980, pp. 15-60.

3. M. Hamana, On linear topological properties of some $C^{*}$-algebras, Tôhoku Math. J. 29 (1977), 157-163.

4. H. Hanche-Olsen and E. Stormer, Jordan operator algebras, Pitman, London, 1984.

5. E. Stormer, Jordan algebras of type I, Acta Math. 115 (1966), 165-184.

6. D. Topping, An isomorphism invariant for spin factors, J. Math. Mech. 15 (1966), 10551063.

Department of Mathematics, Reading University, P.O. Box 220, Reading RG6 2AX, ENGLAND 